Chapman University

Chapman University Digital Commons

$11-26-2020$

Regiospecific Synthesis of Calcium-Independent Daptomycin Antibiotics Using a Chemoenzymatic Method

Nagaraju Mupparapu

Yu-Hsin Cindy Lin

Tae Ho Kim

Sherif I. Elshahawi

Follow this and additional works at: https://digitalcommons.chapman.edu/pharmacy_articles

Part of the Medicinal and Pharmaceutical Chemistry Commons, and the Other Pharmacy and Pharmaceutical Sciences Commons 
Regiospecific Synthesis of Calcium-Independent Daptomycin Antibiotics Using a Chemoenzymatic Method

\section{Comments}

This is the accepted version of the following article:

Mupparapu N, Lin YC, Kim TH, Elshahawi SI. Regiospecific synthesis of calcium-independent daptomycin antibiotics using a chemoenzymatic method. Chemistry. 2020;10.1002/chem.202005100.

which has been published in final form at https://doi.org/10.1002/chem.202005100. This article may be used for non-commercial purposes in accordance with Wiley Terms and Conditions for Self-Archiving.

\section{Copyright}

Wiley 


\section{Chemistry \\ Chemistry Europe}

A European Journal

European Chemical Societies Publishing

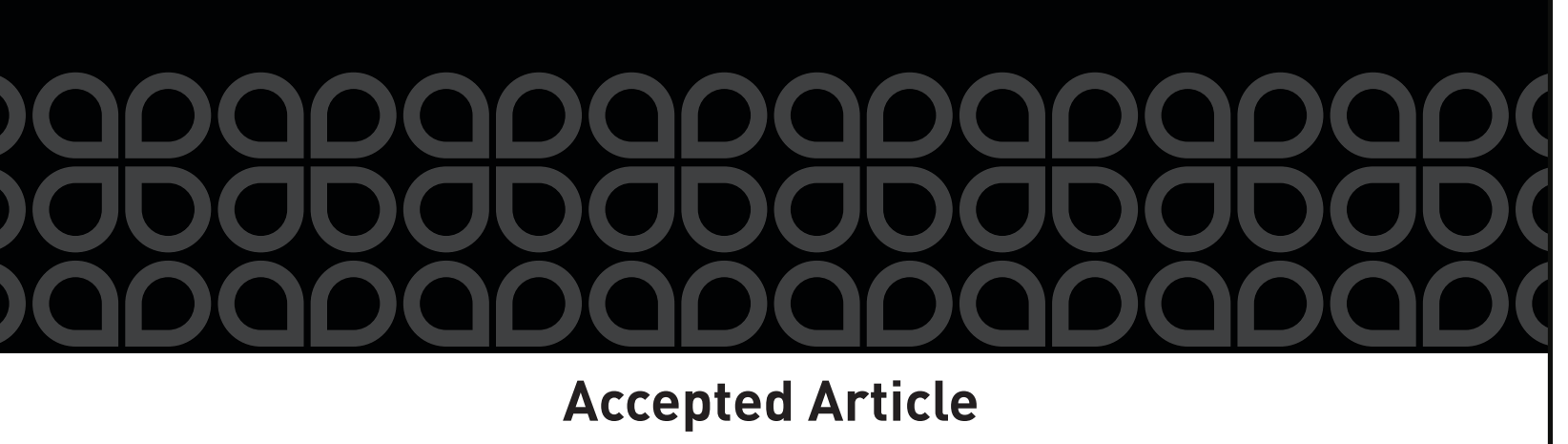

Title: Regiospecific Synthesis of Calcium-Independent Daptomycin Antibiotics Using a Chemoenzymatic Method

Authors: Nagaraju mupparapu, Yu-Hsin Cindy Lin, Tae Ho Kim, and Sherif I Elshahawi

This manuscript has been accepted after peer review and appears as an Accepted Article online prior to editing, proofing, and formal publication of the final Version of Record (VoR). This work is currently citable by using the Digital Object Identifier (DOI) given below. The VoR will be published online in Early View as soon as possible and may be different to this Accepted Article as a result of editing. Readers should obtain the VoR from the journal website shown below when it is published to ensure accuracy of information. The authors are responsible for the content of this Accepted Article.

To be cited as: Chem. Eur. J. 10.1002/chem.202005100

Link to VoR: https://doi.org/10.1002/chem.202005100 
WILEY-VCH

\title{
Regiospecific Synthesis of Calcium-Independent Daptomycin Antibiotics Using a Chemoenzymatic Method
}

\author{
Nagaraju Mupparapu, Yu-Hsin Cindy Lin, Tae Ho Kim, Sherif I. Elshahawi ${ }^{\star[a]}$
}

\author{
[a] Professor SI Elshahawi, Dr. N Mupparapu, Ms Y-H C Lin, Dr. TH Kim \\ Department of Biomedical and Pharmaceutical Sciences \\ Chapman University School of Pharmacy \\ Rinker Health Science Campus \\ Irvine, CA 92618 \\ E-mail: elshahawi@chapman.edu
}

Supporting information for this article is given via a link at the end of the document.

\begin{abstract}
Daptomycin (DAP) is a calcium $\left(\mathrm{Ca}^{2+}\right)$-dependent FDAapproved antibiotic drug for the treatment of Gram-positive infections. It possesses a complex pharmacophore hampering derivatization and/or synthesis of analogs. In order to mimic the $\mathrm{Ca}^{2+}$-binding effect we used a chemoenzymatic approach to modify the tryptophan (Trp) residue of DAP and synthesize kinetically characterized and structurally elucidated regiospecific Trp-modified DAP analogs. We demonstrated that the modified DAPs are several-folds active than the parent molecule against antibiotic-susceptible and antibioticresistant Gram-positive bacteria. Strikingly and in contrast to the parent molecule, the DAP derivatives do not rely on calcium or any additional elements for activity.
\end{abstract}

\section{Introduction}

Daptomycin/Cubicin ${ }^{\circledR}$ (DAP, 1, Fig. 1a) is the latest first-in-class FDA-approved antibiotic drug for the treatment of bacteremia, endocarditis and complicated skin and skin structure infections..$^{[1,2]}$ DAP is the only systemically applied membraneactive antibiotic prescribed for the treatment of Gram-positive bacterial infections. ${ }^{[3-5]}$ Furthermore, DAP has become the last resort drug for the treatment of challenging infections arising from antibiotic-resistant Gram-positive bacteria. ${ }^{[6]}$ DAP is an anionic calcium $\left(\mathrm{Ca}^{2+}\right)$-dependent antibiotic, where $\mathrm{Ca}^{2+}$ plays an essential role in its activity and resistance by decreasing the required concentration for the 1 membrane insertion 50 -fold..$^{[7]}$ Although not fully understood, the DAP mechanism of action (MOA) requires $\mathrm{Ca}^{2+}$ binding resulting in neutralization of the DAP negative charges and in conformational changes involving the aromatic residues L-tryptophan ( $\left.\operatorname{Trp}_{1}\right)$ and kynurenine (Kyn 13$)$. The new conformation exposes 1 hydrophobic regions, increases its amphipathicity and switches the overall charge of 1 to positive enhancing DAP oligomerization. ${ }^{[8,9]}$ It is well accepted that the cationic $\mathrm{DAP} / \mathrm{Ca}^{2+}$ complex targets the anionic lipid phosphatidylglycerol (PG) of the cell membrane leading to either ion leakage or cell membrane lipid extraction and subsequent membrane dissipation and cell death. ${ }^{[7]}$ Recently, a MOA showing that $\mathrm{DAP} / \mathrm{Ca}^{2+}$ targets cell wall precursors through the formation of a tripartite complex with PG has been reported. [10] Thus, we propose that modifying the DAP $\operatorname{Trp}_{1}$ or $\mathrm{Kyn}_{13}$ residues will compensate for the $\mathrm{Ca}^{2+}$-induced conformational changes of 1 leading to similar oligomerization and subsequent bacterial cell death.

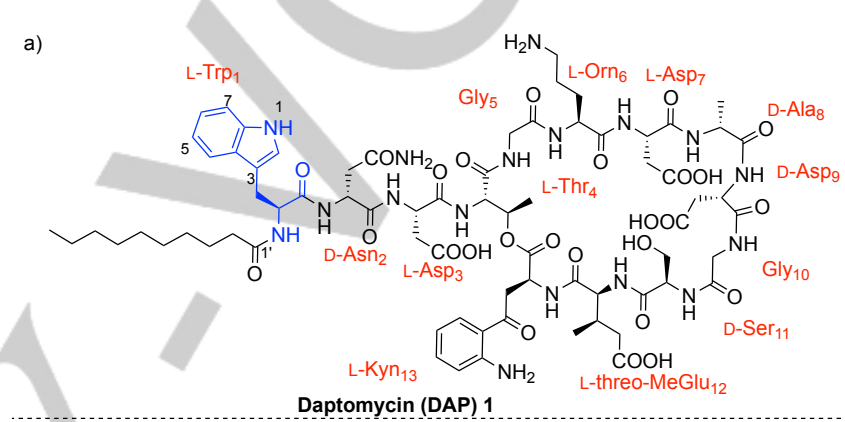

Figure 1. a) Structure of daptomycin (DAP, 1); b) previously reported CdpNPTcatalyzed daptomycin analogs with native donor.

There is a need to develop DAP analogs to overcome its challenges such as incomplete understanding of the MOA, inactivity against pneumonia and rising levels of resistance. However, the structure complexity of $\mathbf{1}$ has delayed efforts to develop analogs. DAP is a cyclic lipopeptide composed of 13 nonproteinogenic amino acids carrying a $n$-decanoic acid chain attached to the amide of L-Trp 1 with multiple chiral centers and reactive groups (Fig. 1a). ${ }^{[11-13]}$ In fact, the most practical approach to supply the market demand for 1 remains fermentation of the producer microorganism, Streptomyces roseosporus and feeding $n$-decanoic acid. In spite of these hurdles, efforts from several groups have led to the development of DAP analogs using synthetic and combinatorial approaches that suffered frequent multistep reactions and/or poor regio- and stereospecficity. ${ }^{[14-17]}$ Data arising from these efforts have generated mostly DAP derivatives with decreased or abolished antibiotic activities. Yet, analogs with modifications on the aromatic acids, especially $\operatorname{Trp}_{1}$, or replacing it with another aromatic amino acid maintained or increased activity consistent with our hypothesis mentioned above. ${ }^{[16,18-20]}$ Biocatalysis has gained much interest lately and has been used to catalyze difficult and uncommon reactions due to its advantage of regio- and stereospecificity. ${ }^{[21-25]}$ Indole prenyltransferases (IPTs) are enzymes that utilize naturallyoccurring allyl pyrophosphate donors (OPP) to catalyze the 
transfer of the prenyl group to Trp/indole or indole-containing acceptor. The significance of IPTs arise from their relaxed acceptor and donor specificity in addition to their ability to catalyze $C-C$ bond formation. ${ }^{[26,27]}$ Among these promiscuous IPTs is CdpNPT, a fungal IPT that has shown broad substrate specificity. It catalyzes normal $\left(C-1^{\prime}\right)$ and/or reverse $\left(C-3^{\prime}\right)$ prenylation of Trpcontaining cyclic dipeptides at $N-1$ or $\mathrm{C}-3$ of the indole, respectively using native and nonnative OPPs. ${ }^{[28-30]}$ In a previous study, CdpNPT catalyzed the prenylation of 1 with native OPP donor, dimethylallyl pyrophosphate (DMAPP) generating two analogs with normal $\mathrm{N}-1$ and reverse $\mathrm{C}-3$ prenylation that had improved antimicrobial activities (Fig. 1b). ${ }^{[19]}$ This suggested that Trp 1 -modified DAP analogs with improved activities can be generated by combining CdpNPT with a chemical platform of noncommercial nonnative OPP with subsequent $C$ alkylation/arylation. Since conformational changes associated with L-Trp 1 has been shown to play a key role in $\mathbf{1}$ activity upon $\mathrm{Ca}^{2+}$ binding, we hypothesized that the chemoenzymatic approach proposed will lead to DAPs that are less dependent on $\mathrm{Ca}^{2+}$. Herein, we report the regiospecific synthesis of three $C-6$ Trp DAP derivatives using alkyl and aryl OPP as donors and CdpNPT as a biocatalyst. The chemical structures were elucidated using HR-ESI-MS and 1- and 2D NMR and their antimicrobial properties were determined. The analogs displayed enhanced antimicrobial properties against DAP-susceptible and DAP-resistant strains with activity that is independent on $\mathrm{Ca}^{2+}$.

\section{Results and Discussion}

Our fundamental goal was to synthesize regiospecific and bioactive Trp 1 -DAP analogs using CdpNPT enzyme combined with synthetic nonnative OPPs (Fig. 2a). Thus, we had the E. colicodon optimized $c d p N P T$ gene synthesized (Supplementary Fig. 1). The gene was cloned into a $\mathrm{pET} 28 \mathrm{a}$ vector and transformed into $E$. coli $5 \alpha$ for sequence verification. The $\mathrm{N}$-His6-CdpNPT (herein referred to as CdpNPT) was heterologously produced in E. coli BL21 (DE3) and purified to homogeneity (Supplementary Fig. 2). In parallel, we synthesized nine alkyl/aryl pyrophosphate (OPP) donors $\mathbf{2} \mathbf{a}-\mathbf{2} \mathbf{i}$ that cover different chemical space containing a double bond at the $\beta$-position to the diphosphate group to be accepted by IPTs (Fig. 2b). ${ }^{[2]}$ Established protocols were followed to generate OPPs by using the corresponding alkyl/aryl bromide $\mathbf{4}$ as precursors treated with tetrabutylammonium pyrophosphate (TBAPP) at room temperature for 2-3 h (Fig. 2b, Supplementary Methods). ${ }^{[31,32]}$ In the case $\mathbf{4}$ was not commercially available or cost prohibitive, the aldehyde was used to generate the desired bromide as in the case of $\mathbf{2 g}$ (Fig. $2 \mathrm{~b}$ ). A series of nonnative donors with allyl (2a, $\mathbf{2 b}, \mathbf{2 c})$, propargyl (2f) cyclic alkenes $(\mathbf{2 g})$ and aromatic $(\mathbf{2 h}, \mathbf{2 i})$ groups were synthesized to demonstrate different functionalities

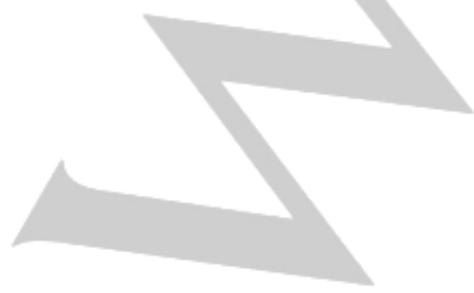

a)

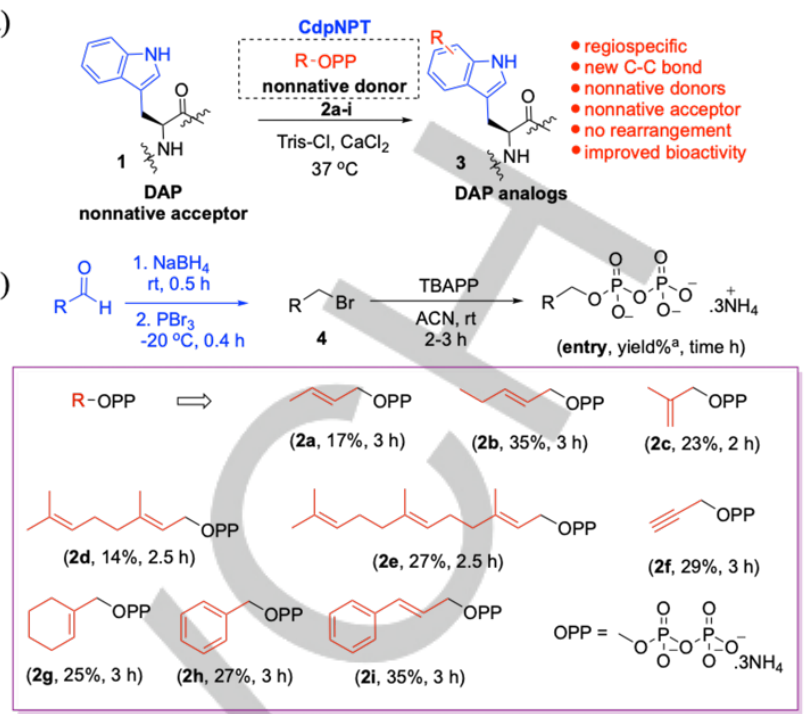

c) 1

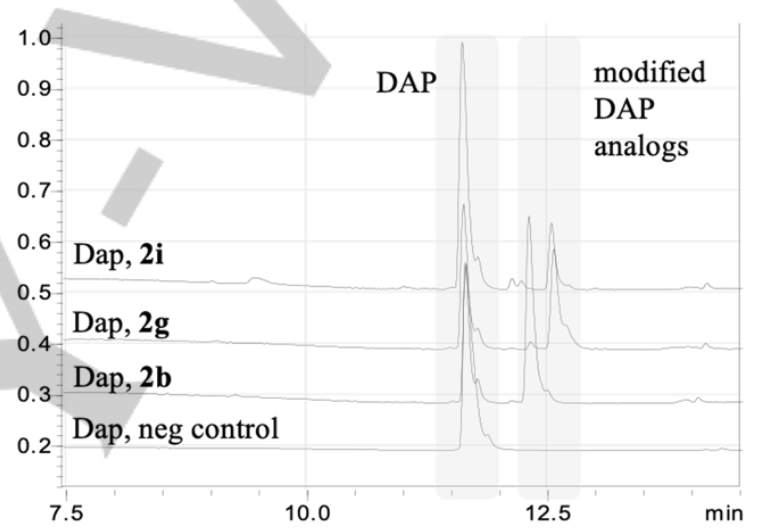

Figure 2. a) CdpNPT-catalyzed reactions of daptomycin analogs $\mathbf{3 x} ; \mathbf{b}$ ) synthesis of pyrophosphate donors 2, aisolated yield after column chromatography; c) HPLC chromatogram of reaction mixture containing daptomycin in Tris $50 \mathrm{mM} / \mathrm{CaCl}_{2} 10 \mathrm{mM}\left(\mathrm{pH} \mathrm{8.0)}\right.$ incubated for $16 \mathrm{~h}$ at $37^{\circ} \mathrm{C}$ in the presence of each of $\mathbf{2 b}, \mathbf{2} \mathbf{g}$ and $\mathbf{2 i}$.

(Fig. 2b). In addition, two analogs were synthesized to mimic the naturally-occurring donors, geranyl diphosphate (GPP, 2d) and farnesyl diphosphate (FPP, 2e). The OPP series included donors with variable chain lengths and two carried terminal alkene (2c) or alkyne (2f) to facilitate future downstream Diels-Alder or $\mathrm{Cu}$ mediated click chemistry, respectively. The purified OPPs were confirmed by HR-ESI-MS, ${ }^{1} \mathrm{H}$ and ${ }^{31} \mathrm{P}$ NMR analyses (Supplementary Methods, Supplementary Mass Spectra, Supplementary NMR Spectra).

To assess the potential of CdpNPT to modify DAP, analytical scale reactions were carried out using $0.4 \mathrm{mM} \mathrm{1,0.8} \mathrm{mM} \mathrm{2a-2i}$ and $16 \mu \mathrm{M}$ CdpNPT in $50 \mathrm{mM}$ Tris buffer (pH 8.0) supplemented with $10 \mathrm{mM} \mathrm{CaCl}_{2}$ at $37^{\circ} \mathrm{C}$ for $16 \mathrm{~h}$. Empty $\mathrm{pET} 28 \mathrm{a}$ E. coli extract and subjected to the same purification method was used as negative control. Analytical scale reactions revealed the formation of a new product in each of the reactions with (E)-pent-2-en-1-yl (2b), cyclohex-1-en-1-ylmethyl (2g) and cinnamyl (2i) diphosphates with conversion percentages of 30,28 and $15 \%$, respectively as observed by HPLC and MS (Fig. 2c, Supplementary Mass Spectra). Furthermore, conversion of $<5 \%$ was also shown with the but-2-en-1-yl (2a) and benzyl (2h) diphosphates as indicated by HPLC and HR-ESI-MS 
(Supplementary Mass Spectra). Thus, CdpNPT was able to diversify 1 with OPPs attached to moieties with 4-9 carbons

a)<smiles>CC/C=C/Cc1ccc2c(C[C@H](NC)C(=O)O)c[nH]c2c1</smiles>

c)

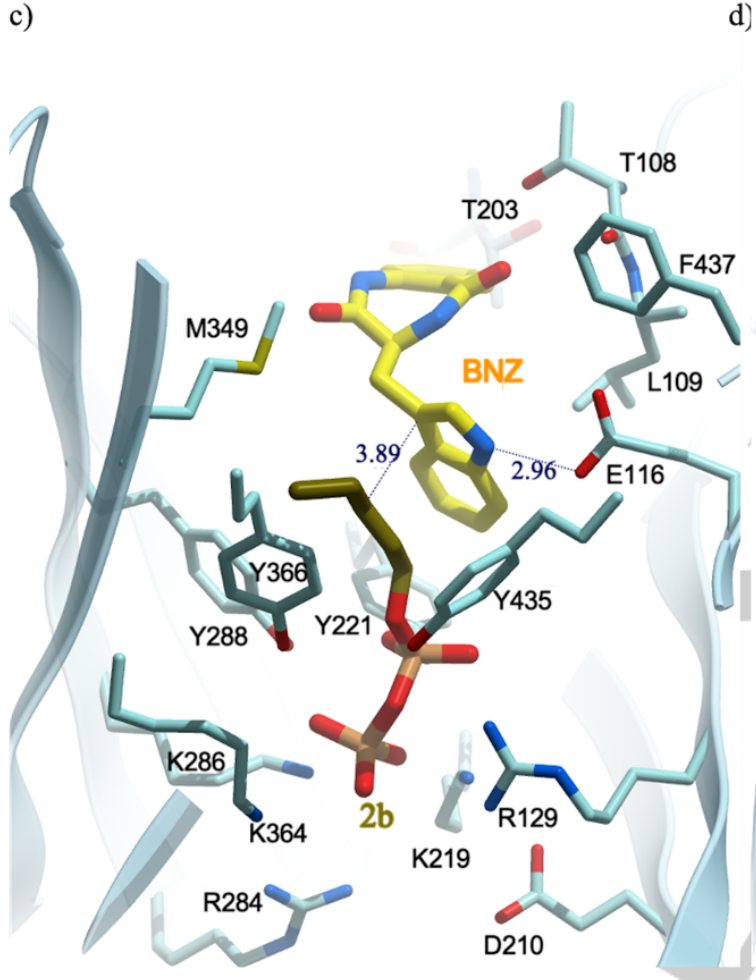

c)

e)

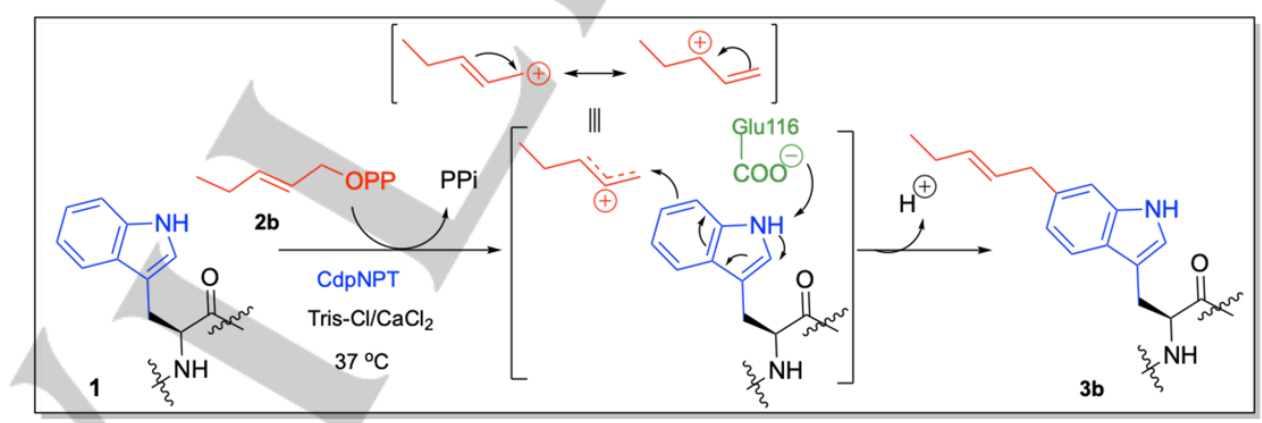

d)

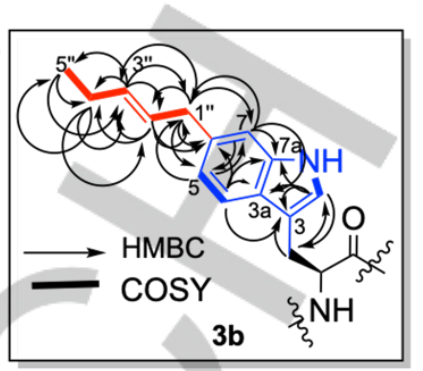

Figure 3. a) Structures of daptomycin analogs $\mathbf{3 b}, \mathbf{3} \mathbf{g}$ and $\mathbf{3 i}$ synthesized enzymatically; b) COSY and HMBC correlations of $\mathbf{3 b}$; c), d) docking models of CdpNPT (PDB ID 4EOU) using (E)-pent-2-en-1-yl diphosphate (2b) as a donor and (S)-indolylmethylbenzodiazependione (c, Bnz) and daptomycin (d, DAP). Hydrogen bonds are shown in as black dashes and their corresponding lengths are given in $\AA$; e) proposed mechanism for the formation of $\mathbf{3 b}$.

including alkyl and aromatic groups. All positive reactions generated one major single product based on chromatograms (Fig. 2b).

Michaelis-Menten kinetic parameters of CdpNPT for all three alkyl/aryl OPPs $(\mathbf{2 b}, \mathbf{2 g}, \mathbf{2} \mathbf{i})$ with DAP were determined. The kinetic parameters, Michaelis constant $\left(K_{m}\right)$ as well as turnover number $\left(k_{\mathrm{cat}}\right)$ were determined from a nonlinear regression fit of initial velocities versus the concentration of the alkyl/aryl OPPs by GraphPad Prism 8.4.3 (Supplementary Table 1). Moreover, we studied the kinetic parameters of $\mathbf{2 b}$ with cyclo-L-Trp-L-Tyr (Supplementary Fig 3a), a commercially available Trp-containing dipeptide that structurally mimics the native acceptor $S$ - 
indolylmethylbenzodiazependione (BNZ, Supplementary Fig 3b), and has shown to act as a substrate for CdpNPT (Supplementary Figs. 3-6). ${ }^{[3,34]}$ Subsequent determination of CdpNPT steadystate kinetic parameters of $\mathbf{1 / 2 b}$ (Supplementary Fig. 3) and cyclo(L-Trp-L-Tyr)/2b (Supplementary Fig. 4) showed the catalytic efficiency to be $0.76 \mathrm{M}^{-1} \mathrm{~s}^{-1}$ and $0.93 \mathrm{M}^{-1} \mathrm{~s}^{-1}$, respectively (Supplementary Table 1). This shows that CdpNPT can accommodate both acceptors with the same donor in similar rates, in spite of the significant size differences. As expected, and consistent with our HPLC initial screening, the catalytic efficiencies of CdpNPT with $1 / 2 \mathrm{~g}\left(k_{\text {cat }} / K_{\mathrm{m}} \approx 0.72 \mathrm{M}^{-1} \mathrm{~s}^{-1}\right)$ (Supplementary Fig. 5) was comparable to that of $\mathbf{1 / 2}$ b. CdpNPT with DAP/2i had the lowest catalytic efficiency $\left(k_{\text {cat }} / K_{\mathrm{m}} \approx 0.39 \mathrm{M}^{-1}\right.$ $\mathrm{s}^{-1}$ ) (Supplementary Fig. 6). This proves that CdpNPT can accommodate alkyl substrates with moderate carbon chain length $(5-7)$ in a similar fashion but efficiency decreases when the chain length of the OPP donor is longer due to potential steric hindrance in the enzyme active site.

To assess the regiospecificity of CdpNPT with DAP and each of $\mathbf{2 b}, \mathbf{2} \mathbf{g}$ and $\mathbf{2} \mathbf{i}$, the reactions were scaled up. Reactions in Tris 50 $\mathrm{mM}$ (pH 8.0) supplemented with $10 \mathrm{mM} \mathrm{CaCl}_{2}$, containing 1 (0.8$2 \mathrm{mM})$, OPP (1.4-2 mM) and $24 \mu \mathrm{M}$ CdpNPT were prepared and incubated at $37^{\circ} \mathrm{C}$ for $16 \mathrm{~h}$. Preparative RP-HPLC purification led to single modified DAP from each reaction, $\mathbf{3 b}, \mathbf{3} \mathbf{g}$ and $\mathbf{3 i}$ with isolated yields of $22 \%, 21 \%$ and $15 \%$, respectively (Supplementary Methods). For structural elucidation purified DAP analogs were subjected to HR-ESI-MS and 1- and 2D NMR spectroscopy to determine regiospecificity (Fig. 3a, Supplementary Fig. 7). For $3 \mathbf{b}$, the (+)-HR-ESI-MS $[\mathrm{M}+\mathrm{H}]^{+} \mathrm{m} / \mathrm{z}$ 1688.7761 indicated a molecular formula of $\mathrm{C}_{77} \mathrm{H}_{109} \mathrm{~N}_{17} \mathrm{O}_{26}$ (Supplementary Mass Spectra). The MS spectrum shows that $\mathbf{3 b}$ has an additional $\Delta m / z=68$ compared to 1 consistent with an additional $-\mathrm{C}_{5} \mathrm{H}_{8}-$ group. The ${ }^{1} \mathrm{H},{ }^{13} \mathrm{C}$ and $\mathrm{HSQC}$ NMR spectroscopic data revealed 77 signals attributable to 5 methyl, 23 methylene, 23 methine, and 26 nonprotonated carbons (Supplementary Table 2, Supplementary NMR Spectra). Comparison of $3 b{ }^{1} \mathrm{H}$ and ${ }^{13} \mathrm{C}$ NMR data with $1{ }^{1} \mathrm{H}$ and ${ }^{13} \mathrm{C}$ NMR data indicated the presence of additional 1 methyl $\delta_{\mathrm{C}} 13.8$ (C-5"), 2 methylene $\delta_{\mathrm{C}} 38.6(\mathrm{C}-1 "), \delta_{\mathrm{C}} 24.9$ (C-4") and 2 methine, $\delta_{\mathrm{C}}$ 129.0 (C-2"), $\delta_{\mathrm{C}} 132.2$ (C-3"). ${ }^{1} \mathrm{H}-{ }^{1} \mathrm{H}$ COSY correlations between $\mathrm{H}-1 "\left(\delta_{\mathrm{H}} 3.34\right) / \mathrm{H}-2$ " ( $\left.\delta_{\mathrm{H}} 5.56\right), \mathrm{H}-3$ " $\left(\delta_{\mathrm{H}} 5.52\right) / \mathrm{H}-4$ " ( $\left.\delta_{\mathrm{H}} 1.99\right)$ and $\mathrm{H}-4 " / \mathrm{H}-5$ " $\left(\delta_{\mathrm{H}} 0.94\right)$ as well as the ${ }^{1} \mathrm{H}-{ }^{13} \mathrm{C} \mathrm{HMBC}$ correlations $(\mathrm{H}-$ 5" to $\mathrm{C}-3$ ", $\mathrm{H}-4$ " to $\mathrm{C}-3$ "/C-2" and $\mathrm{H}-1$ " to $\mathrm{C}-3$ "/C-2") deduced the presence of a pent-2-en-1-yl group indicating the presence of a normal (C-1") not reverse (C-3") reaction (Fig. 3b, Supplementary Fig. 8). All of the remaining COSY and HMBC correlations of $\mathbf{3 b}$ were in full agreement with that of 1 except for the indole moiety (Supplementary Table 2, Supplementary NMR Data). The COSY correlations between $\mathrm{H}-4\left(\delta_{\mathrm{H}} 7.47\right) / \mathrm{H}-5\left(\delta_{\mathrm{H}} 6.79\right)$ and $\mathrm{H}-1\left(\delta_{\mathrm{H}}\right.$ $10.62) / \mathrm{H}-2\left(\delta_{\mathrm{H}} 7.06\right)$ as well as the HMBC correlations between $\mathrm{H}-5$ to $\mathrm{C}-1 " / \mathrm{C}-7$ ( $\left.\delta_{\mathrm{C}} 110.5\right), \mathrm{H}-7$ ( $\left.\delta_{\mathrm{H}} 7.07\right)$ to $\mathrm{C}-1 " / \mathrm{C}-5$ ( $\left.\delta_{\mathrm{c}} 119.3\right)$, $\mathrm{H}-2$ " to $\mathrm{C}-6$ ( $\delta \mathrm{c}$ 133.2) and $\mathrm{H}-1$ " to $\mathrm{C}-5 / \mathrm{C}-7$ determined the regiospecificity to be at the $C-6$ of DAP indole. The configurational assignments of $2 "-E$ were deduced by calculating the coupling constants of $\mathrm{H}-2$ " ( $\mathrm{dt}, \mathrm{J}=15.2,6.0 \mathrm{~Hz}$ ). Thus, the structure of $\mathbf{3 b}$ was established to be 6-C-((E)-pent-2-en-1-yl)-L-Trp 1 -daptomycin For structure elucidation of $\mathbf{3 g}$ and $\mathbf{3 i}$, the HR-ESI-MS $[\mathrm{M}+\mathrm{H}]^{+}$ $\mathrm{m} / \mathrm{z} 1714.7959$ and $\mathrm{m} / \mathrm{z} 1736.7820$ indicated the molecular formulas of $\mathrm{C}_{79} \mathrm{H}_{111} \mathrm{~N}_{17} \mathrm{O}_{26}$ and $\mathrm{C}_{81} \mathrm{H}_{110} \mathrm{~N}_{17} \mathrm{O}_{26}$, respectively. 1- and 2D NMR established $\mathbf{3 g}$ and $\mathbf{3 i}$ to be $6-C$-(cyclohex-1-en-1ylmethyl)-L-Trp 1 -daptomycin and 6-C-(cinnamyl)-L-Trp 1 - daptomycin, respectively (for full structure elucidation of $\mathbf{3 g}$ and 3i, see Supplementary Methods, Supplementary Fig. 9, Supplementary Fig. 10, Supplementary Mass Spectra and Supplementary NMR Spectra). This highlights the significance of this chemoenzymatic approach in developing regiospecific DAP analogs at a challenging position as the indole $C-6$. During the preparation of this manuscript, Scull et al. reported the generation of DAP analogs $\mathbf{3 g}$ and $\mathbf{3 i}$ using CdpNPT. ${ }^{[35]}$ However, our kinetically-characterized reactions led to regiospecific DAP products in contrast to the reported work which could be attributed to the increase in enzyme promiscuity under different conditions. ${ }^{[36]}$ Noteworthy, comparing the analogs synthesized herein $(C-6)$ with those generated from previous efforts shows that changing the donors used with DAP/CdpNPT system leads to variation in the alkylation/arylation positions of $1 .{ }^{[19,35]}$

We sought to investigate the structural basis of DAP docking into the binding pocket of CdpNPT and determine the basis of regiospecific DAP analogs produced in this study and discrepancy when compared to native acceptor. CdpNPT catalyzes the reverse prenylation of BNZ and cyclo-L-Trp-L-Tyr at the C-3 using DMAPP (native donor) and $\mathbf{2 b}$ respectively, which rearranges to provide the normal prenyl at $N-1 .{ }^{[30,32,34,37]}$ Indeed, CdpNPT catalyzed the nonnative DAP prenylation at the $N-1$ and C-3 positions when coupled with DMAPP (Fig. 1b). ${ }^{[19]}$ Since the $3 \mathrm{D}$-structure of a DAP-CdpNPT-bound complex is not available, we used molecular modeling of the ternary CdpNPT 3D-structure cocrystallized with the native acceptor BNZ and a donor mimic, thiolodiphosphate (PDB ID 4EOU). ${ }^{[37]}$ Thus, ICM Molsoft was used to virtually dock $\mathbf{2 b}$ with each of BNZ (Fig. 3c) and DAP (Fig. 3d). The binding pocket was identified and the hits with the lowest score indicated less energy and higher stability. Our models show that the donor $\mathbf{2 b}$ is anchored in the binding pocket by electrostatic interaction between the anionic $\mathbf{2 b}$ diphosphate and the cationic residues (Arg129, Lys219, Arg284, Lys286, Lys364) in addition to a hydrogen bond with Tyr431. ${ }^{[3]}$ Previous reports support that the formed carbocation, generated from the loss of the diphosphate, is stabilized by the tyrosine shield (Tyr 221, Tyr288, Tyr366, Tyr431) via $\pi$-cation interactions. ${ }^{[26,37]}$ It is expected that $\mathbf{2 g}$ will exist in the chair form inside the cavity taking a comparable size as $\mathbf{2} \mathbf{b}$ which could explain the similar catalytic efficiencies. Compared to $\mathbf{2} \mathbf{g}, \mathbf{2} \mathbf{h}$ produced less yield which could be explained by flattening of the aromatic ring and subsequent steric hindrance. Replacing the methyl group in $\mathbf{2} \mathbf{b}$ with phenyl in $2 i$ led to decrease in the enzyme kinetics showing the effect of steric hindrance on the catalytic efficiency. The cavity surrounding the donor $\mathbf{2 b}$ is spacious enough to allow for slightly larger chain such as the cinnamyl group in 2i. The residue Met349 seems to act as the gatekeeper preventing CdpNPT from accepting larger donors. Indeed, engineering of M349G has provided a biocatalyst that can accommodate GPP (2d) when combined with a simple dipeptide as acceptor. ${ }^{[33]}$ This implies that using the engineered CdpNPT_M349G instead of wild-type enzyme with $\mathbf{1}$ is a promising approach to diversify DAP when coupled with bulkier donors.

The indole-moiety of the acceptor is positioned between the alkyl chain of OPP and Glu116 whose role is to act as a nucleophile to activate the indole ring (Fig. 3c, Fig. 3d). In our model, the $\mathrm{NH}$ and C-3 of the BNZ are distanced at 2.96 and $3.89 \AA$ from the Glu116 acidic and the C-3 alkyl side chain of $\mathbf{2 b}$, respectively (Fig. $3 \mathrm{C}$ ). On the other hand, 1 fits itself deep onto the spacious binding pocket of CdpNPT where the indole faces downward parallel to 
the attached $n$-decanoic lipid chain. DAP docks such that it is positioned at $3.20 \AA$ and $3.42 \AA$ from the Glu116 acidic side chain and $\mathrm{C}-1$ of the $\mathbf{2 b}$ alkyl side chain, respectively with $300^{\circ}$ clockwise rotation compared to the BNZ indole (Fig. 3d). This orientation allows for access of the OPP C-1" to attack the $C-6$ of the DAP Trp. DAP seem to form hydrophobic interactions with the nonpolar regions of 1 with the residues Leu109, Ala131, Val201, Val206, Val207, Ala208, Phe223, Phe272, Trp419 and form hydrogen bond with polar residues including Cys107, Thr108, Thr203, Asp210 and Glu220. Hence, the proposed mechanism of the formation of these analogs is initiated by the release of the diphosphate from the donor (Fig. 3e). Subsequent Friedel-Crafts aromatic substitution at the $C-6$ position of the indole leads to the formation of an arenium intermediate followed by deprotonation to restore aromaticity and produce the $C$-6-substituted DAP analogs. ${ }^{[26,27]}$

Table 1. MIC values of daptomycins DAP, $\mathbf{3 b}, \mathbf{3 g}$, and $\mathbf{3 i}$, in the presence of $50 \mathrm{mg} \mathrm{L}^{-1}$ calcium and $10 \mathrm{mM}$ EDTA, respectively. NG, no growth; values in $\mu \mathrm{M}$ (mg $\mathrm{L}^{-1}$ ). Daptomycin-resistant strains are highlighted in green. The most well established daptomycin-resistant mechanisms include DAP-repulsion or diversion away from the bacterial division septum. This involves the reduction of the anionic PG content by downregulating the PG synthase ( $p g s A$ ), lysinylation of $P G$ via MprF increase in the cardiolipin synthesis on the expense of PG, shedding of the PG lipids or D-alanylation of teichoic acids. ${ }^{[3,6,38]}$

\begin{tabular}{|c|c|c|c|c|c|c|c|c|c|}
\hline \multirow[b]{2}{*}{ Type } & \multirow[b]{2}{*}{ Strain } & \multicolumn{4}{|c|}{$\mathrm{Ca}^{2+}$} & \multicolumn{4}{|c|}{ EDTA } \\
\hline & & DAP & $3 b$ & $3 \mathbf{g}$ & $3 \mathbf{i}$ & DAP & $3 b$ & $3 \mathbf{g}$ & $3 \mathbf{i}$ \\
\hline \multirow{15}{*}{$\begin{array}{c}\text { Gram } \\
\text { positive }\end{array}$} & E. faecalis B-537 & $3.60(5.8)$ & $0.25(0.4)$ & $0.38(0.6)$ & $0.45(0.8)$ & $>72(>116)$ & $1.00(1.7)$ & $0.75(1.3)$ & $0.90(1.6)$ \\
\hline & DAP-susc. E. faecalis $\mathrm{S} 613$ & $3.60(5.8)$ & $2.00(3.4)$ & $1.50(2.6)$ & $1.80(3.1)$ & $36(58)$ & $2.00(3.4)$ & $1.50(2.6)$ & $1.80(3.1)$ \\
\hline & DAP-res. E. faecalis R712 & $18(29)$ & $2.00(3.4)$ & $1.50(2.6)$ & $1.80(3.1)$ & $>72(>116)$ & $4.00(6.8)$ & $3.10(5.3)$ & $1.80(3.1)$ \\
\hline & E. faecium TX1330 & $9.0(14.5)$ & $1.00(1.7)$ & $0.75(1.3)$ & $0.90(1.6)$ & $>72(>116)$ & $2.00(3.4)$ & $1.50(2.6)$ & $1.80(3.1)$ \\
\hline & E. faecium UAA714 & $9.0(14.5)$ & $1.00(1.7)$ & $0.75(1.3)$ & $0.90(1.6)$ & $72(116)$ & $2.00(3.4)$ & $1.50(2.6)$ & $1.80(3.1)$ \\
\hline & S. aureus ATCC 6538 & $1.8(2.9)$ & $0.50(0.85)$ & $0.75(1.3)$ & $0.90(1.6)$ & $36(58)$ & $1.00(1.7)$ & $0.75(1.3)$ & $0.90(1.6)$ \\
\hline & Methicillin res. S. aureus & $1.8(2.9)$ & $0.50(0.85)$ & $0.75(1.3)$ & $0.90(1.6)$ & $36(58)$ & $2.00(3.4)$ & $1.50(2.6)$ & $1.80(3.1)$ \\
\hline & Vancomycin res. S. aureus & $1.8(2.9)$ & $0.50(0.85)$ & $0.75(1.3)$ & $0.45(0.8)$ & $72(116)$ & $2.00(3.4)$ & $1.50(2.6)$ & $1.80(3.1)$ \\
\hline & S. aureus SA-1199B & $1.8(2.9)$ & $0.50(0.85)$ & $0.38(0.6)$ & $0.45(0.8)$ & $72(116)$ & $2.00(3.4)$ & $1.50(2.6)$ & $1.80(3.1)$ \\
\hline & S. aureus SAUSA3001713 & $9(14.5)$ & $0.5(0.85)$ & $0.75(1.3)$ & $0.90(1.6)$ & $>72(>116)$ & $2.00(3.4)$ & $1.50(2.6)$ & $1.80(3.1)$ \\
\hline & S. aureus SAUSA3001715 & $9(14.5)$ & $0.5(0.85)$ & $0.75(1.3)$ & $0.90(1.6)$ & $>72(>116)$ & $2.00(3.4)$ & $1.50(2.6)$ & $1.80(3.1)$ \\
\hline & Micrococcus luteus B-287 & $0.22(0.4)$ & $0.06(0.1)$ & $0.19(0.33)$ & $0.23(0.4)$ & $3.60(5.8)$ & $0.25(0.4)$ & $0.19(0.3)$ & $0.23(0.4)$ \\
\hline & Bacillus subtilis B-14596 & $1.80(2.9)$ & $0.12(0.2)$ & $0.09(0.15)$ & $0.23(0.4)$ & $72(116)$ & $0.50(0.9)$ & $0.38(0.6)$ & $0.45(0.8)$ \\
\hline & Mycobacterium aurum & $>36(>58)$ & $>40(>67)$ & $>31(>53)$ & $>18(>31)$ & NG & NG & NG & NG \\
\hline & Mycobacterium smegmatis & $>36(>58)$ & $>40(>67)$ & $>31(>53)$ & $>18(>31)$ & NG & NG & NG & NG \\
\hline \multirow{3}{*}{$\begin{array}{c}\text { Gram } \\
\text { negative }\end{array}$} & Enterobacter cloacae & $>36(>58)$ & $>40(>67)$ & $>31(>53)$ & $>18(>31)$ & $>36(>58)$ & $>40(>67)$ & $>31(>53)$ & $>18(>31)$ \\
\hline & Salmonella enterica & $>36(>58)$ & $>40(>67)$ & $>31(>53)$ & $>18(>31)$ & $>36(>58)$ & $>40(>67)$ & $>31(>53)$ & $>18(>31)$ \\
\hline & Escherichia coli & $>36(>58)$ & $>40(>67)$ & $>31(>53)$ & $>18(>31)$ & $>36(>58)$ & $>40(>67)$ & $>31(>53)$ & $>18(>31)$ \\
\hline Fungus & Saccharomyces cerevisiae & $>36(>58)$ & $>40(>67)$ & $>31(>53)$ & $>18(>31)$ & $>36(>58)$ & $>40(>67)$ & $>31(>53)$ & $>18(>31)$ \\
\hline
\end{tabular}

Prenylated DAP analogs modified with native donor DMAPP have shown improved antimicrobial properties when compared to the parent $1{ }^{[19]}$ Thus, we screened our purified analogs with different bacterial and fungal strains (Table 1). No activity was detected in any of the modified analogs against the Gram-negative bacteria Enterobacter cloacae UCI 3, Salmonella enterica, or Escherichia coli or the fungus Saccharomyces cerevisiae (Table 1). Furthermore, the analogs showed no increase in cytotoxicity against HEK293 eukaryotic cells when compared to 1 . Our preliminary screening assays using DAP and $\mathbf{3 b}$ were tested against a small panel of Gram-positive bacteria in the presence of $50 \mathrm{mg} \mathrm{L}^{-1} \mathrm{Ca}^{2+}$ as recommended for antimicrobial screening of lipopeptides in general and DAP in specific (Supplementary Table 4). ${ }^{[39,40]}$ Analog 3 b showed $4-33$ fold MIC improvement when compared to 1 . In order to assess the reliance of generated DAPs on $\mathrm{Ca}^{2+}$, we repeated the screening in the absence of any added ions. As expected, we found that DAP MIC deteriorated by 2-30 fold. In fact, the MIC even increased several folds in the absence of the chelating agent EDTA which is expected as $\mathrm{Ca}^{2+}$ is essential for $\mathbf{1}$ activity. Strikingly, $\mathbf{3} \mathbf{b}$ was minimally affected by the absence of $\mathrm{Ca}^{2+}$ (Supplementary Table 4). In order to ensure that no undetected impurities are present due to purification process which might contribute to activity, we used unreacted 1 that was incubated in enzymatic reactions with CdpNPT and $\mathbf{2 b}$ in the presence of buffer and was purified via reversed-phase HPLC as a positive control. We obtained comparable results showing that at least one of our enzymatically-synthesized compounds, not DAP, is active in the absence of $\mathrm{Ca}^{2+}$. To confirm our results and to test a broader aspect on antimicrobial activities, we subjected our three Trp 1 -modified analogs, in addition to 1 to a final HPLC run to get rid of any residual acid from the initial purification that might contribute to activity, as indicated by lack of peaks corresponding to formic acid or trifluoro acetic acid in our ${ }^{1} \mathrm{H}$ and ${ }^{13} \mathrm{C}$ NMR spectra. The concentrations of the tested materials were then adjusted by measuring absorbance using Beer-Lambert law. We then repeated the antimicrobial screening using all purified analogs subjected to similar conditions to a wider panel of 19 microorganisms of different antibiotic-resistant bacteria including the three DAP-resistant strains $E$. faecalis R712, S. aureus SAUSA300_1713 and S. aureus SAUSA300_1715 (Table 1). In the presence of $50 \mathrm{mg} \mathrm{L}^{-1} \mathrm{Ca}^{2+}$, analogs $3 \mathbf{b}, \mathbf{3 g}$ and $3 \mathbf{i}$ possessed 1.5-20 fold improved MIC when compared to 1 against Grampositive strains including methicillin, vancomycin and ciprofloxacin-resistant strains. The three analogs possessed comparable MICs to each other with $3 \mathbf{b}$ being slightly better (Table 1). DAP forms a cationic complex with $\mathrm{Ca}^{2+}$ (Fig. 4a) that 
causes conformational changes involving $\operatorname{Trp}_{1}$ and ion charge variations. This allows 1 to bind the anionic lipid membranes, possibly at the bacterial division septum with subsequent membrane dissipation (Fig. 4c). ${ }^{[7,8]}$ Interestingly, while DAPresistant $E$. faecalis $\mathrm{R} 712$ was 5 -fold more tolerant to 1 than DAPsusceptible $E$. faecalis $\mathbf{S} 613$, the enzymatically-modified analogs (Fig. 4b) were equally active against both strains highlighting the importance of Trp ${ }_{1}$-modification to combat resistance (Table 1). Similar results were seen with the two DAP-resistant $S$. aureus strains (Table 1). In the absence of $\mathrm{Ca}^{2+}$ via the addition of EDTA as a chelating agent, $\mathbf{1}$ activity was deteriorated or abolished (Table 1). On the contrary, the $\mathbf{3} \mathbf{b}, \mathbf{3} \mathbf{g}$ and $\mathbf{3} \mathbf{i}$ showed no or minimal effect on MIC suggesting that these analogs avoid the MOA used by DAP/Ca ${ }^{2+}$ complex. DAP-resistance has been postulated to occur via repulsion where the bacterium overproduces cationic lysyl-PG on the expense of anionic PG (Fig. 4d). ${ }^{[7]}$ Diversion is an alternative mechanism which involves anionic membrane lipids acting as DAP sink holes that migrate away from the vital cell division septum. Our $\mathrm{Ca}^{2+}$-independent Trp-modified DAP seem to overcome resistance by avoiding the formation of cationic complex and thus circumvent DAP-repulsion or diversion (Fig. 4d). It is tempting to assume that the biocatalytic modification of the DAP Trp 1 with alkyl or aromatic groups changes the conformation of 1 in a similar fashion when bound to $\mathrm{Ca}^{2+}$ leading to the enhanced insertion of 1 into the cell membrane (Fig. 4c). An alternative explanation where the Trp-modification of 1 led to increase in hydrophobicity and facilitated oligomerization has been suggested but would not explain the impressing activity against DAP-resistant strains and the $\mathrm{Ca}^{2+}$ independency. ${ }^{[16]}$ Taken together, these data suggest that the generated $\mathbf{3 b}, \mathbf{3} \mathbf{g}$ and $3 \mathbf{i}$ analogs act as drug leads with a new MOA that is yet to be explored. a)

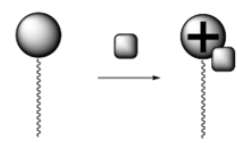

c)

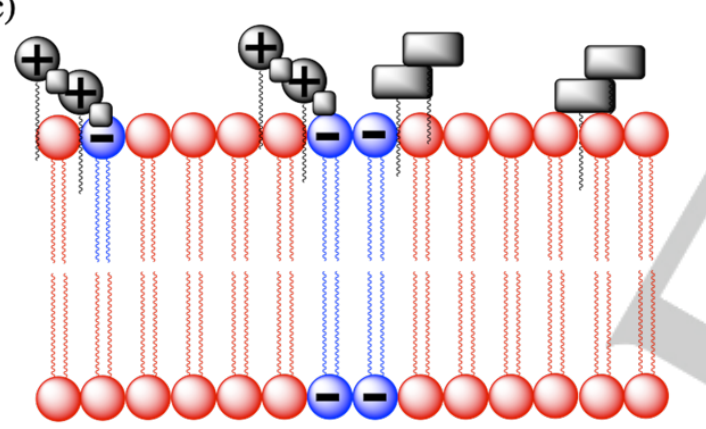

b)

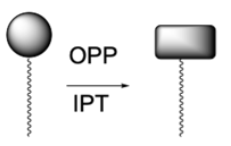

d)

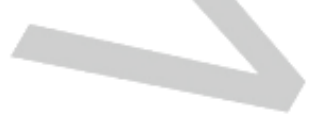

Figure 4. a) Formation of DAP/Ca $\mathrm{C}^{2+}$ complex; b) formation of Trp ${ }_{1}$-modified daptomycin analogs (3b, $\mathbf{3 g}$ and $\left.\mathbf{3 i}\right)$; c) proposed mechanisms of actions for DAP/Ca ${ }^{2+}$ and $\mathbf{3 b}, \mathbf{3 g}$ and $\mathbf{3 i}$ rely on binding to the anionic lipids located at the bacterial division septum leading to DAP or DAP analogs oligomerization and subsequent membrane dissipation; d) Proposed mechanism for $\mathbf{3 b} \mathbf{3} \mathbf{3} \mathbf{g}$ and $\mathbf{3 i}$ to overcome DAP-resistance. Bacteria develop DAP-resistance by decreasing the anionic lipid content or via diversion od DAP to other areas away from the division septum. Trp-modified DAP analogs are capable of binding to the neutral or cationic lipids causing bacterial growth inhibition. OPP, alkyl and aryl pyrophosphate; IPT, indole pyrophosphate; ML, membrane lipid; AL, anionic lipids such as phosphatidylglycerol; LPG, lysylphosphatidylglycerol; DAP, daptomycin.

\section{Conclusion}

We synthesized a library of diverse alkyl and aryl OPPs (2a-2i) and coupled them with a promiscuous IPT, CdpNPT to generate regiospecific $C$ - 6 Trp1-modified DAP derivatives via $C$ - $C$ bond formation. The kinetically-characterized compounds were scaled up, purifed and the structures were determined using MS and 1and $2 \mathrm{D}$ NMR spectroscopy. The DAP analogs $\mathbf{3 b}, \mathbf{3 g}$ and $\mathbf{3 i}$ were found to be several folds active than the parent 1 against different antibiotic-resistant Gram-positive bacteria with no apparent effect on cytotoxicity. Moreover, these analogs will feed into the DAPanalogs pipeline to fully reveal the 1-MOA as well as the DAPresistance mechanism. Most importantly, $\mathbf{3 b}, \mathbf{3 g}$ and $\mathbf{3 i}$ were active in the absence of $\mathrm{Ca}^{2+}$, an essential ion for DAP and other
$\mathrm{Ca}^{2+}$-dependent antibiotic lipopeptides, which is unprecedented. Yet, further studies are needed to explain how the enzymatically synthesized DAP derivatives inhibit Gram positive bacteria in the absence of $\mathrm{Ca}^{2+}$. The current study opens the door for drug properties enhancement for cationic antimicrobial peptides that works through the same MOA of DAP/Ca ${ }^{2+}$ complex. Furthermore, this work provides a proof-of-concept for the regiospecific diversification and differential alkylation/arylation of $\mathbf{1}$ and other Trp-containing peptides for drug discovery and other synthetic/tagging applications using wild-type and/or engineered IPTs.

\section{Acknowledgements}


This work was supported by the New Investigator Award from the American Association of Colleges of Pharmacy and Chapman University School of Pharmacy. We are grateful for undergraduate students Zhengfeiyue (Chelsea) Pan, Lauren Brewster, Tiffany Nguyen, Katrina Ostrom, Christopher Nguyen and Nikita Dave for assistance in enzyme overproduction and purification as well as screening assays. We are thankful for Dr. Innokentiy Maslennikov and the Nuclear Magnetic Resonance facility at CUSP. The microbial organisms used in screening were obtained through BEI Resources, NIAID, NIH.

\section{Conflict of Interest}

The authors declare no competing financial interest.

Keywords: antibiotics • biological activity • enzymes • mechanism of action $\cdot$ natural products

[1] P. Kirkpatrick, A. Raja, J. LaBonte, J. Lebbos, Nat. Rev. Drug Discov. 2003, 2, 943-944.

[2] V. G. Fowler, H. W. Boucher, G. R. Corey, E. Abrutyn, A. W. Karchmer, M E. Rupp, D. P. Levine, H. F. Chambers, F. P. Tally, G. A. Vigliani, C. H. Cabell, A. S. Link, I. DeMeyer, S. G. Filler, M. Zervos, P. Cook, J. Parsonnet, J. M. Bernstein, C. S. Price, G. N. Forrest, G. Fätkenheuer, M. Gareca, S. J. Rehm, H. R. Brodt, A. Tice, S. E. Cosgrove, S. aureus Endocarditis and Bacteremia Study Group, N. Engl. J. Med. 2006, 355, 653665.

[3] D. A. Gray, M. Wenzel, Antibiot. Basel Switz. 2020, 9, DOI 10.3390/antibiotics 9010017 .

[4] J. G. Hurdle, A. J. O'Neill, I. Chopra, R. E. Lee, Nat. Rev. Microbiol. 2011 9, $62-75$.

[5] C. Ghosh, J. Haldar, ChemMedChem 2015, 10, 1606-1624

[6] T. T. Tran, J. M. Munita, C. A. Arias, Ann. N. Y. Acad. Sci. 2015, 1354, 32 53.

[7] W. R. Miller, A. S. Bayer, C. A. Arias, Cold Spring Harb. Perspect. Med. 2016, 6, DOI 10.1101/cshperspect.a026997.

[8] S. W. Ho, D. Jung, J. R. Calhoun, J. D. Lear, M. Okon, W. R. P. Scott, R. E. W. Hancock, S. K. Straus, Eur. Biophys. J. EBJ 2008, 37, 421-433.

[9] D. Jung, A. Rozek, M. Okon, R. E. W. Hancock, Chem. Biol. 2004, 11, 949 957.

[10] F. Grein, A. Müller, K. M. Scherer, X. Liu, K. C. Ludwig, A. Klöckner, M. Strach, H.-G. Sahl, U. Kubitscheck, T. Schneider, Nat. Commun. 2020, 11, 1455 .

[11] M. Debono, M. Barnhart, C. B. Carrell, J. A. Hoffmann, J. L. Occolowitz, B. J. Abbott, D. S. Fukuda, R. L. Hamill, K. Biemann, W. C. Herlihy, J. Antibiot. (Tokyo) 1987, 40, 761-777.

[12] V. Miao, M.-F. Coëffet-Legal, P. Brian, R. Brost, J. Penn, A. Whiting, S. Martin, R. Ford, I. Parr, M. Bouchard, C. J. Silva, S. K. Wrigley, R. H. Baltz, Microbiol. Read. Engl. 2005, 151, 1507-1523.

[13] M. Debono, B. J. Abbott, R. M. Molloy, D. S. Fukuda, A. H. Hunt, V. M. Daupert, F. T. Counter, J. L. Ott, C. B. Carrell, L. C. Howard, J. Antibiot. (Tokyo) 1988, 41, 1093-1105.

[14] K. T. Nguyen, D. Ritz, J.-Q. Gu, D. Alexander, M. Chu, V. Miao, P. Brian, R. H. Baltz, Proc. Natl. Acad. Sci. U. S. A. 2006, 103, 17462-17467.

[15] R. H. Baltz, ACS Synth. Biol. 2014, 3, 748-758.

[16] H. Y. Chow, K. H. L. Po, P. Gao, P. Blasco, X. Wang, C. Li, L. Ye, K. Jin, K. Chen, E. W. C. Chan, X. You, R. Yi Tsun Kao, S. Chen, X. Li, J. Med. Chem. 2020, 63, 3161-3171.

[17] C. R. Lohani, R. Taylor, M. Palmer, S. D. Taylor, Org. Lett. 2015, 17, 748 751.

[18] H. Y. Chow, K. H. L. Po, K. Jin, G. Qiao, Z. Sun, W. Ma, X. Ye, N. Zhou, S Chen, X. Li, ACS Med. Chem. Lett. 2020, DOI 10.1021/acsmedchemlett.0c00175.

[19] S. I. Elshahawi, H. Cao, K. A. Shaaban, L. V. Ponomareva, T. Subramanian, M. L. Farman, H. P. Spielmann, G. N. Phillips, J. S. Thorson, S. Singh, Nat. Chem. Biol. 2017, 13, 366-368.

[20] Y. He, J. Li, N. Yin, P. S. Herradura, L. Martel, Y. Zhang, A. L. Pearson, V. Kulkarni, C. Mascio, K. Howland, J. A. Silverman, D. D. Keith, C. A. Metcalf, Bioorg. Med. Chem. Lett. 2012, 22, 6248-6251.

[21] C. E. Boville, R. A. Scheele, P. Koch, S. Brinkmann-Chen, A. R. Buller, F. H. Arnold, Angew. Chem. Int. Ed. 2018, 57, 14764-14768.

[22] Y. Yang, I. Cho, X. Qi, P. Liu, F. H. Arnold, Nat. Chem. 2019, 11, 987-993.

[23] Y. Zhang, J. Zhang, L. V. Ponomareva, Z. Cui, S. G. Van Lanen, J. S. Thorson, J. Am. Chem. Soc. 2020, 142, 9389-9395.

[24] S. I. Elshahawi, T. A. Ramelot, J. Seetharaman, J. Chen, S. Singh, Y. Yang, K. Pederson, M. K. Kharel, R. Xiao, S. Lew, R. M. Yennamalli, M. D Miller, F. Wang, L. Tong, G. T. Montelione, M. A. Kennedy, C. A. Bingman, H. Zhu, G. N. Phillips, J. S. Thorson, ACS Chem. Biol. 2014, 9, 2347-2358. H.-L. Ruan, W.-B. Yin, J.-Z. Wu, S.-M. Li, ChemBioChem 2008, 9, 1044

[31] V. J. Davisson, A. B. Woodside, T. R. Neal, K. E. Stremler, M. Muehlbacher, C. D. Poulter, J. Org. Chem. 1986, 51, 4768-4779.

[32] M. Liebhold, X. Xie, S.-M. Li, Org. Lett. 2012, 14, 4882-4885.

[33] G. Liao, P. Mai, J. Fan, G. Zocher, T. Stehle, S.-M. Li, Org. Lett. 2018, 20 , 7201-7205.

[34] M. Liebhold, X. Xie, S.-M. Li, Org. Lett. 2013, 15, 3062-3065.

[35] E. M. Scull, C. Bandari, B. P. Johnson, E. D. Gardner, M. Tonelli, J. You, R. H. Cichewicz, S. Singh, Appl. Microbiol. Biotechnol. 2020, 104, 7853-7865. [36] I. Nobeli, A. D. Favia, J. M. Thornton, Nat. Biotechnol. 2009, 27, 157-167.

[37] J. M. Schuller, G. Zocher, M. Liebhold, X. Xie, M. Stahl, S.-M. Li, T. Stehle, J. Mol. Biol. 2012, 422, 87-99.

[38] C. M. Ernst, A. Peschel, Int. J. Med. Microbiol. IJMM 2019, 309, 359-363.

[39] I. Wiegand, K. Hilpert, R. E. W. Hancock, Nat. Protoc. 2008, 3, 163-175.

[40] R. H. Baltz, in Nat. Prod. Chem. Drug Discov., RSC Publishing, 2009, pp. 395-409.

. Arnold, Angew. Chem. Int. Ed Engl. 2018, 57, 4143-4148.

W.-B. Yin, H.-L. Ruan, L. Westrich, A. Grundmann, S.-M. Li,

ChemBioChem 2007, 8, 1154-1161.

. Arnold, Angew Che 2015, 32,88-101.
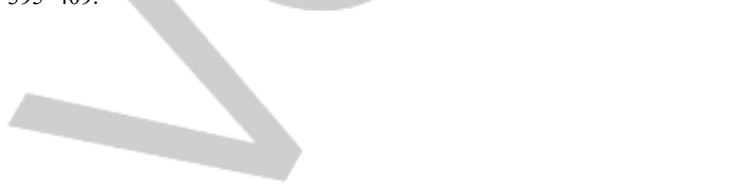

(




\section{Entry for the Table of Contents}
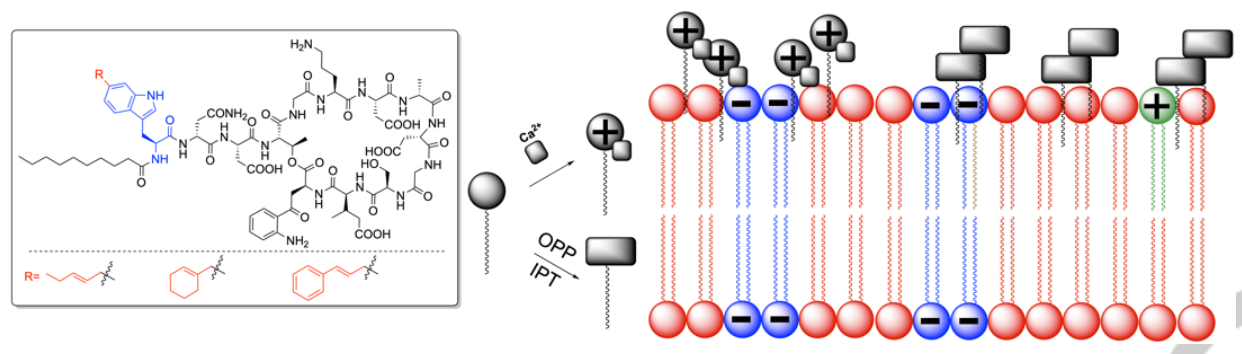

Daptomycin relies on calcium as an essential element for its strong antibiotic activity. Herein, we use a chemoenzymatic approach to synthesize regiospecific daptomycin derivatives that are independent on calcium and are more active than the parent daptomycin against daptomycin-susceptible and daptomycin-resistant Gram-positive bacteria.

Researcher Twitter username: SherifElshahawi
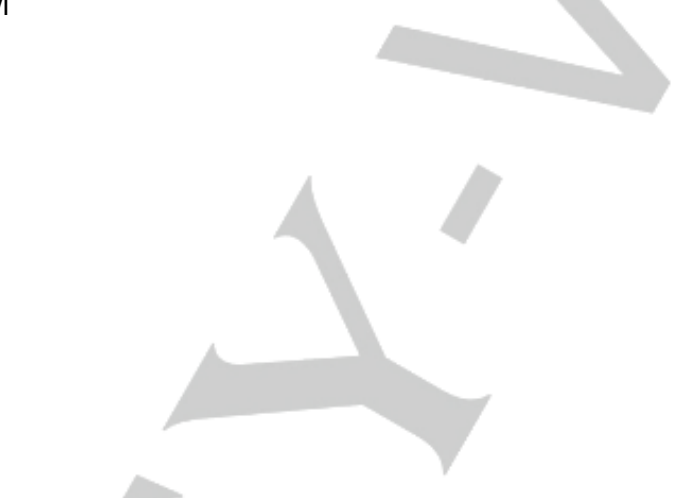